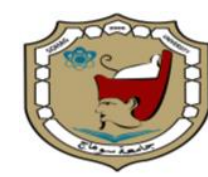

Sohag University

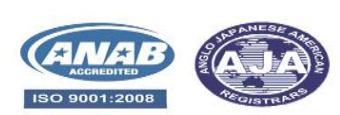

Sohag Medical Journal

Faculty of Medicine

\title{
Feasibility And Effectiveness Of Retrosigmoid Approach In Vestibular Schwannoma Surgery
}

\author{
Mohamed Awad Mohamed *, Walid Khalaf Abouzeid *, Mohamed \\ Ahmed Abdelaal ${ }^{*}$, Magda Mohamed Ali ${ }^{* *}$. \\ * Department of Neurosurgery - Faculty of Medicine - Sohag University \\ ** Department of Public Health and Community Medicine, Faculty of Medicine, \\ Sohag University
}

\begin{abstract}
:
Purpose: To assess the feasibility and effectiveness of the lateral suboccipital retrosigmoid approach in the surgical management of vestibular schwannoma.

Materials and Methods: We conducted a prospective hospital-based case series conducted on 356 patients during the period from May 2018 to May 2020 in collaboration with the neurosurgery department, "Sohag University hospital", and "Tokyo Medical University Hospital". Patients included who were undergoing surgical intervention for vestibular schwannoma (VS) through retrosigmoid approach. All patients in our study were followed up for a minimum of four months. For each patient intraoperative tumor resection volume was recorded, postoperative complications, facial and cochlear nerve functions were assessed.

Results: Enrolled in this study 356 cases, the majority $(54.8 \%)$ of patients were females; while (45.2\%) were males were included with a mean age $(42.57 \pm 12.3)$ years. We achieved a mean follow-up of 6.2 months; ranging (4-9 months). Postoperative scoring of facial nerve function was a good outcome in $79.4 \%$, fair outcome in $17.5 \%$, and poor outcome in $3.1 \%$.

Conclusion: We assessed the results of surgery using a retrosigmoid approach in 356 patients with VS. Surgical management of VS using retrosigmoid approach is safe and effective but after the advancement of microsurgical techniques like drilling and neurophysiological monitoring especially for seventh and eighth cranial nerves, the possibility of preserving the facial and cochlear functions became more feasible with excellent results.
\end{abstract}

Key Words: Vestibular Schwannoma, facial, retrosigmoid, Acoustic neuroma.

\section{Introduction}

Vestibular schwannoma (VS) is an extra-axial, benign, encapsulated, Schwann cell-derived tumor, which originates from the vestibular portion of eighth nerves usually within the internal auditory canal (IAC). As the tumor grows into the cerebellopontine angle (CPA), it compresses the cochlear, vestibular, and facial nerves (FN), as well as the cerebellum and brain stem, giving rise to various symptoms. ${ }^{[1][2]}$
Treatment options are: follow up, radiosurgery, and microsurgical excision, depending on the tumor size, location and rate of growth, and hearing level in the affected ear, age, and general health condition also have a great role in managing option. [2]

Surgical approaches used for this intervention include the middle cranial fossa, translabyrinthine, and lateral suboccipital retrosigmoid approaches. The g- 
oal of surgery is changed from mere tumor removal to functional outcome through the concept of the maximum achievable amount of tumor removal avoiding serious persistent postoperative FN palsy. Of the surgical approaches utilized, the retrosigmoid approach has several advantages. Surgery via this approach can be easily performed in individuals with large tumors and those with concurrent hydrocephalus. This approach provides simple and direct access to CPA lesions, with surgical exposure extending from the tentorium and trigeminal nerve superiorly to the foramen magnum and inferiorly to the jugular for amen. Thus, compared with other approaches, the lateral suboccipital retrosigmoid technique provides a better view of the CPA and allows adequate identification of the CPA structures, more complete excision of lesions, fewer complications, and avoids the risks of major neurological complications. ${ }^{[3][4]}$

\section{Subjects and Methods}

We conducted a prospective hospitalbased case series on 356 patients during the period from May 2018 to May 2020 in collaboration with the neurosurgery department, "Sohag University hospital", and "Tokyo Medical University Hospital". Patients admitted for surgical intervention with a diagnosis of VS through a retrosigmoid approach were included. All patients were followed up for at least four months postoperatively. The study was approved by the "Ethics Committee" of our institute.

Among the clinical parameters evaluated were presenting symptoms, pre-and postoperative hearing status using puretone audiometry results, tumor size on MRI scans, and pre-and postoperative assessments of FN function. Tumor size was defined as the magnitude of the longest diameter of the cisternal part of the tumor and graded according to "Koos classification system ${ }^{[5]}$ " into grade II $(\leq$ $2 \mathrm{~cm})$, grade III $(\leq 3 \mathrm{~cm})$, and grade IV
( $>3 \mathrm{~cm}$ ). For each patient, intraoperative tumor resection volume was recorded, as well as postoperative complications. The hearing was graded according to the guidelines of "American Academy of Otolaryngology-Head and Neck Surgery" (AAO-HNS) ${ }^{[6]}$; where classes A \& B (A: $\leq 30, \mathrm{~B}:>30, \leq 50)$ "dB" defined serviceable hearing, classes $\mathrm{C}$ \& $\mathrm{D}(\mathrm{C}:>50 \mathrm{~dB}, \mathrm{D}$ : any other level $)$ non-serviceable hearing according to pure tone audiogram results. FN functions were described using House-Brickman (HB) ${ }^{[7]}$ where "HB Grades I-II" considered good outcome, "HB Grades III-IV" fair, and "HB Grade V-VI" considered poor outcome.

The lateral suboccipital retrosigmoid approach is the standard approach we used. With the patient in the lateral position "Mayfield" pin fixation system was used to fix the head [Figure 1]. The skin incision we used is a curved semilunar on the post-auricular area. After that a craniotomy is initiated with three burr holes, exposing both the transverse-sigmoid sinuses (TS-SS) junction; where the keyhole of our approach is placed. We use a single point on the three-dimensional computed tomography image on the venous phase [Figure 2]. The dural incision is performed in a U-shaped fashion on the bottom of the sigmoid sinus allowing anterior reflection of the dura [Figure 3]. We identify the course of the FN on the tumor surface by an interrupted FN stimulating probe. We came to a point when the tumor could be internally debulked and the walls of the tumor could be collapsed inward. At this stage, the tumor could be dissected away from the cerebellu$\mathrm{m}$, the flocculus, and finally the brainstem and cranial nerves. Then, a continuous FN stimulating probe could be placed upon the root entry zone of the FN. This allowed for real-time FN monitoring during the whole procedure. 
The IAC is drilled laterally (about 6 and $9 \mathrm{~mm}$ out of the posterior IAC wall can be removed) with the limiting structures being the semi-circular ducts and the vestibule laterally and the jugular bulb inferiorly. Longitudinal opening of the IAC dura is carried out and the vestibular nerves are identified. Dissection of the FN from the tumor carried out using smooth tipped forceps. At least temporarily we tried to preserve the cochlear nerve in every case to avoid bearing the FN the full weight of the remaining tumor. At the time when the tumor is completely removed, the field is irrigated and inspected for residual tumor tissues. The closure started with sealing the IAC to prevent a CSF leak. Then, dural closure was reinforced with tissue glue, and three mini-titanium plates were used to replace the bone flap.

\section{Results}

Enrolled in this study 356 cases, the majority $(54.8 \%)$ of patients were females; while $(45.2 \%)$ were males were included with a mean age $(42.57 \pm 12.3)$ years. A follow-up margin of 6.2 months; ranging (4-9 months) was achieved.

At admission; (64.6\%) of patients showed up with hearing impairment, $(73.3 \%)$ presented with tinnitus, (53.7\%) presented with face numbness, $(2.2 \%)$ presented with imbalance, $(57.6 \%)$ presented with dizziness or vertigo, $(6.7 \%)$ presented incidentally, (1.4\%) presented with facial paralysis, and $(1.1 \%)$ presented with trigeminal $\mathrm{n}$ euralgia [Table 1].

\begin{tabular}{|l|l|c|}
\hline \multicolumn{2}{|l|}{ Variables } & Frequency (\%) \\
\hline \multicolumn{2}{|l|}{ Duration of symptoms (months) } & $8(1-36) *$ \\
\hline \multirow{3}{*}{ Presenting (initial) symptoms } & Hearing loss & $230(64.6 \%)$ \\
\cline { 2 - 3 } & Tinnitus & $261(73.3 \%)$ \\
\cline { 2 - 3 } & Face numbness & $191(53.7 \%)$ \\
\cline { 2 - 3 } & Imbalance & $8(2.2 \%)$ \\
\cline { 2 - 3 } & Dizziness or vertigo & $205(57.6 \%)$ \\
\cline { 2 - 3 } & Incidental & $24(6.7 \%)$ \\
\cline { 2 - 3 } & Facial paralysis & $4(1.4 \%)$ \\
\cline { 2 - 3 } & Trigeminal neuralgia & $4.1 \%)$ \\
\hline
\end{tabular}

Table 1: Presenting (initial) symptoms among 356 Vestibular Schwannoma patients: *Median (IQR: interquartile range) b
Right-sided tumor presented in 170 patients $(47.8 \%)$, and left-sided in 181 patients $(50.8 \%)$, and bilateral in 5 patients (1.4\%). Regarding tumor size 72 $(20.2 \%)$ was classified as grade II, 123 $(34.6 \%)$ as grade III, and $161(45.2 \%)$ as grade IV.

Regarding preoperative hearing assessment, $(29.9 \%)$ of patients were graded as $\mathrm{A},(15.8 \%)$ were graded $\mathrm{B},(2 \%)$ were graded $\mathrm{C}$, and $(52.4 \%)$ were graded $\mathrm{D}$ according to AAO-HNS hearing classification.

Regarding preoperative FN function; $(96.9 \%)$ of patients had good function (HB I \& II), (2\%) had fair function (HB III \& IV), and (1.1\%) had poor function (HB V \& VI).

Postoperative FN function; (79.4\%) of patients had good function (HB I \& II), (17.5\%) had fair function (HB III \& IV), and $(3.1 \%)$ had poor function (HB V \& VI) [Table 2].

The average resection ratio was (96 \pm $4.5) \%$ in the whole study group; with $(85.9 \%)$ of patients had near-total resection (more than $95 \%$ of tumor size), and $(14.1 \%)$ had subtotal resection (less than $95 \%$ of tumor size).

Postoperative complications included persistent FN dysfunction in 10 patients $(2.8 \%)$, intracranial hemorrhage in 5 (1.4\%), CSF leakage through either wound leak or rhinorrhea $(9.7 \%)$, and 6 (1.6\%) patients who developed wound infection which could be managed conservatively. 
SOHAG MEDICAL JOURNAL

Vol. 25 No. 2 April 2021
Feasibility And Effectiveness Of Retrosigmoid Approach Mohamed Awad Mohamed

\begin{tabular}{|c|c|c|c|}
\hline FN function (HB) & Pre-operative & Post-operative & $\begin{array}{c}\text { Chi-square test } \\
\text { "P value" }\end{array}$ \\
\hline HB-1 & $332(93.8 \%)$ & $231(65.3 \%)$ & \multirow{6}{*}{$<0.0001 * *$} \\
\hline HB-2 & $11(3.1 \%)$ & $50(14.1 \%)$ & \\
\hline HB-3 & $6(1.7 \%)$ & $43(12.1 \%)$ & \\
\hline HB-4 & $1(0.3 \%)$ & $19(5.4 \%)$ & \\
\hline HB-5 & $3(0.8 \%)$ & $8(2.3 \%)$ & \\
\hline HB-6 & $1(0.3 \%)$ & $1(0.3 \%)$ & \\
\hline
\end{tabular}

Table 2: Comparison between pre and postoperative facial nerve function using Chi-square test

*Good (HB Grade I-II), Fair (HB Grade III-IV), Poor (HB Grade V-VI)

** Percentage of Column Total

\begin{tabular}{|l|c|c|}
\hline \multirow{2}{*}{\multicolumn{1}{|c|}{ Associated Factor }} & \multicolumn{2}{c|}{ Post-operative HB grade } \\
\cline { 2 - 3 } & r & P \\
\hline Age & 0.2063 & $=0.0001^{* *}$ \\
\hline Size of tumor & 0.1889 & $=0.0004^{* *}$ \\
\hline IAM extension & 0.007037 & $=0.8952$ \\
\hline PTA (Degree of hearing loss) & 0.1547 & $=0.0077^{*} *$ \\
\hline Duration of symptoms & 0.09972 & $=0.0929$ \\
\hline Resection ratio & -0.3001 & $<0.0001^{*} *$ \\
\hline
\end{tabular}

Table 3: Pearson's correlation analysis for some pre-operative Factors associated with postoperative facial nerve function

$r$ : Pearson's rho (correlation coefficient)

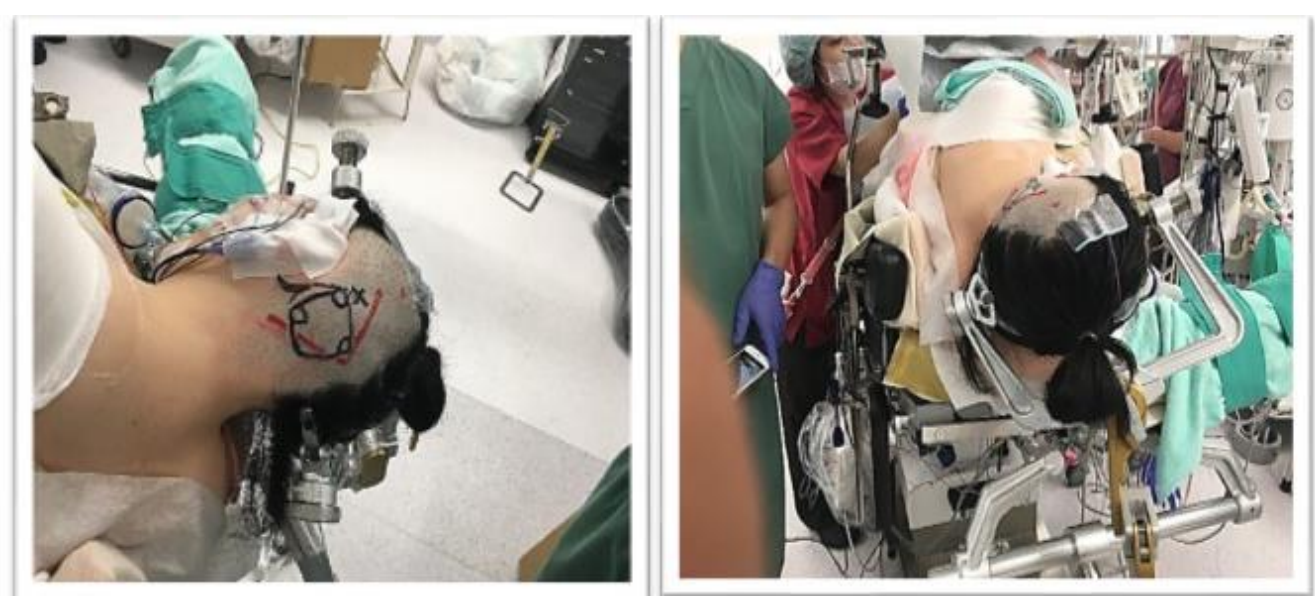

Figure 1: Patient positioning and skin markings

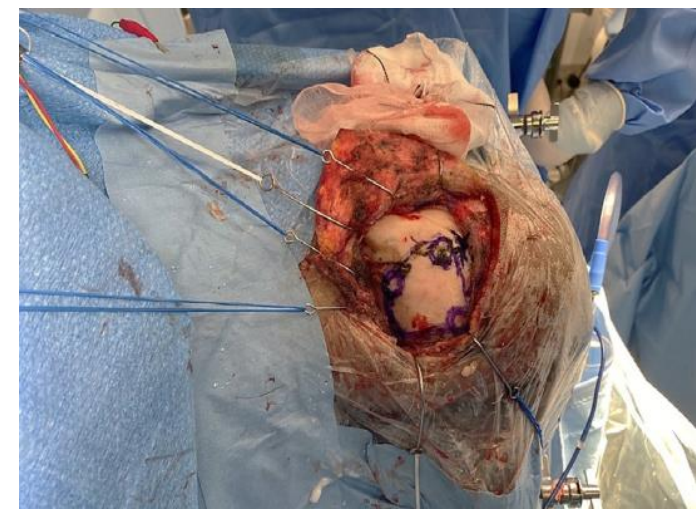

Figure 2: Markings for craniotomy

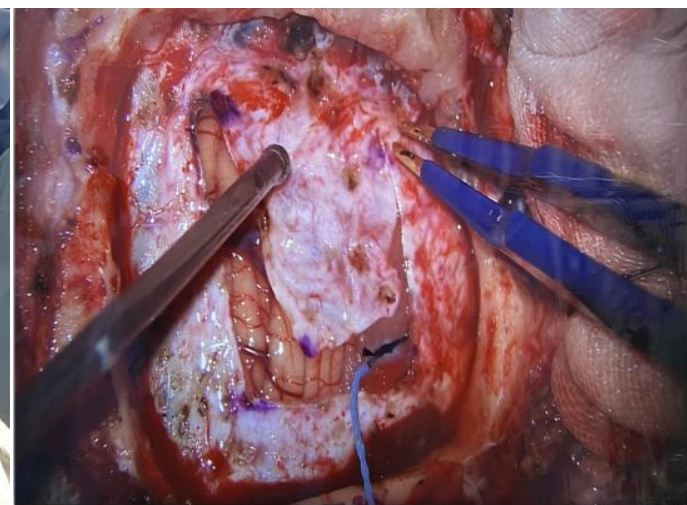

Figure 3: Intraoperative photograph demonstrating U-shaped dural opening 


\section{Discussion}

VS accounts for about $8 \%$ of all intracranial tumors and about $87 \%$ of cerebellopontine angle tumors. ${ }^{[8]}$ These tumors are managed by observation, stereotactic radiosurgery, or microsurgical excision. The surgery is performed using either retrosigmoid, translabyrinthine, or middle fossa approaches. Stereotactic radiosurgery is indicated for the treatment of tumors measuring $\leq 3 \mathrm{~cm}$ in diameter. Surgery is indicated for tumors inadequately controlled by stereotactic radiosurgery. ${ }^{[3]}$

The technique implying the middle cranial fossa approach is used to remove tumors confined to the IAC, especially to preserve hearing ability. Anatomically this approach is technically difficult and carries a high risk of facial palsy. The translabyrinthine approach can also be used, but it carries several disadvantages, especially when removing largesized schwannomas; moreover, most ENT surgeons are more familiar with this approach. The translabyrinthine approach has been recommended, regardless of tumor size, for patients with unserviceable hearing. [9]

A retrosigmoid surgical approach is used by most neurosurgeons; as they are more familiar with the anatomical structures, even with large-sized tumors or in patients with VS associated with hydrocephalus. This approach enables the surgeon to minimize damage to adjacent tissues. However, the location of the FN can't be confirmed unless the tumor is almost completely removed, this approach is associated with a higher risk of FN injury. The retrosigmoid approach is associated with a high incidence of postoperative headache. This approach is used in patients with serviceable hearing, and to remove more medially located tumors. [9]

We preferred the use of the retrosigmoid approach; based on several considerations, including avoidance of the risk of major neurological complications, better postoperative FN function, and hearing preservation.

When we reviewed the major symptoms in our patient group, we found that tinnitus $(73.3 \%)$ was the most common presenting symptom, followed by hearing loss $(64.6 \%)$. That came per studies that have reported that the major symptom of VS is tinnitus, followed by sudden hearing loss, or that smaller-sized tumors were accompanied most frequently by dizziness. In contrast, other previous studies reported that $94 \%$ of patients with VS complained of hearing disturbance. [10][11][12]

Surgery performed via the retrosigmoid approach is advantageous in preserving hearing function in patients with smallsized tumors. In our study, however, abnormal findings were observed in patients with large tumors, $54.4 \%$ of whom had an unserviceable hearing before surgery. [13]

Typically, patients are placed in a lateral or sitting/semi-sitting position for surgery. In our study, we used only the lateral position during surgery. On the other hand, many surgeons in previous publications favored the sitting/semisitting position for this type of surgery. [14][15][16][17] But, the semi-sitting position is associated with a higher risk of air embolism, despite anesthetic precautions to prevent this complication. ${ }^{[18][19][20]}$ Also, the semi-sitting position is associated with a higher incidence of hematoma following excision of cystic schwannoma, ${ }^{[14]}$ whereas the incidence of hematomas that occurred when patients were in the lateral position, was very $\mathrm{u}$ ncommon. On the other hand, the use of lateral position resulted in intraoperative bleeding from peritumoral veins may, that should be managed with meticulous hemostasis. [21][22]

The average resection ratio was $(96 \pm$ $4.5) \%$ in our study group; with $(85.9 \%)$ 
of patients had near-total resection (more than $95 \%$ of tumor size), and (14.1\%) had subtotal resection (less than $95 \%$ of tumor size). As we attempted to preserve the facial nerves, hearing ability, and major intracranial structures as possible as we could. Management of patients with residual tumors included either regular follow-up with MRI or stereotactic radiosurgery.

We found that overall postoperative functions of the FN were good because preoperative $\mathrm{FN}$ dysfunction isn' $\mathrm{t}$ a common presentation of VS. We found that $96.9 \%$ of patients with good preoperative FN function; preservation of function after surgery was achieved in $(79.4 \%)$ of patients with good function (HB I \& II), and (17.5\%) with fair function. Thus, only $(3.1 \%)$ of our patients had poor function (HB V \& VI), a lower percentage than previously observed. [23] In our study group, only ten patients (2.8\%) had permanent FN dysfunction. In literature, VS tumors excision via a translabyrinthine [24][25][26] or retrosigmoid [16][14][27] approach preserved FN function in $70-80 \%$ of patients, with $42-$ $52.6 \%$ showing good function. ${ }^{[23][24][28]}$ The degree of recovery from FN palsy has been defined as good (HB grade I/II) or fair (HB grade III/IV). Preservation of $\mathrm{FN}$ function requires intraoperative monitoring using a continuous $\mathrm{FN}$ SE. [29]

Among the postoperative complications of the retrosigmoid approach for exciseon of VS are death, hemorrhage in the CPA and cerebellum, brain stem injury, post-operative hydrocephalus, CSF leakage, meningitis, cerebellar infarction, lower cranial nerves dysfunction, FN injury, cerebellar edema, intra-operative cardiac arrest, trigeminal dysfunction, abducens dysfunction, diplopia, $\mathrm{u}-$ rinary tract infection, bronchial infection, and diabetic deregulations. However, we found that intracranial hemorrhage happened in $5(1.4 \%)$ of our patients; among which three patients needed reoperation to evacuate the hematoma, and two managed conservatively. CSF leakage through either wound leak or rhinorrhea occurred in $(9.7 \%)$, and six patients $(1.6 \%)$ developed wound infection which could be managed conservatively. ${ }^{[3][30]}$

\section{Conclusion}

We assessed the results of surgery using a retrosigmoid approach in 356 patients with VS. Surgical management of VS using retrosigmoid approach is safe and effective but after the advancement of microsurgical techniques like drilling and neurophysiological monitoring especially for seventh and eighth cranial nerves, the possibility of preserving the facial and cochlear functions became more feasible with excellent results.

\section{References}

1. Khrais T, Romano G, Sanna M. Nerve origin of vestibular schwannoma: a prospective study. J Laryngol Otol. 2007;122(2):128-31.

2. Lin EP, Crane BT. The Management and Imaging of Vestibular Schwannomas. AJNR Am J Neuroradiol. 2017;38(11):2034-43.

3. Briggs RJS, Fabinyi G, Kaye AH. Current management of acoustic neuromas: Review of surgical approaches and outcomes. J Clin Neurosci. 2000;7(6):521-6.

4. Heller RS, Silveira L, Heilman CB. Cerebellopontine Angle Tumors. In: Principles of Neurological Surgery. 2018.

5. Koos WT, Lang J. Tumors of the cerebellopontine angle. Color atlas Microneurosurg York Thieme Med Publ. 1993;510-608.

6. Monsell EM. American Academy of Otolaryngology-Head and Neck Surgery (AAO-HNS) Committee on Hearing and Equilibrium guidelines for the diagnosis and evaluation of therapy in Ménière's disease. Otolaryngol Head Neck Surg. 1995;113:181-5. 
7. House WE. Facial nerve grading system. Otolaryngol Head Neck Surg. 1985;93:184-93.

8. Mahaley Jr MS, Mettlin C, Natarajan N, Laws Jr ER, Peace BB. Analysis of patterns of care of brain tumor patients in the United States: a study of the Brain Tumor Section of the AANS and the CNS and the Commission on Cancer of the ACS. Clin Neurosurg. 1990;36:347.

9. Nuseir A, Sequino G, De Donato G, Taibah A, Sanna M. Surgical management of vestibular schwannoma in elderly patients. Eur Arch Oto-RhinoLaryngology. 2012;269(1):17-23.

10.Park KH, Choung YH, Kim CH, Lee WS. Clinical Evaluation of Small Vestibular Schwannoma. Korean J Otolaryngol Neck Surg. 2016;47(1):226.

11.Mann W, Gouveris HT. Diagnosis and therapy of vestibular schwannoma. Expert Review of Neurotherapeutics. 2009.

12.Rosahl S, Bohr C, Lell M, Hamm K, Iro $\mathrm{H}$. Diagnostics and therapy of vestibular schwannomas - an interdisciplinary challenge. GMS Curr Top Otorhinolaryngol Head Neck Surg. 2017;16.

13. Tan LA, Gerard CS, Ahuja SK, Moftakhar R. Retrosigmoid approach for resection of cerebellopontine angle meningioma and decompression of the trigeminal nerve. Neurosurg Focus. 2014;36(1).

14. Samii M, Matthies C. Management of 1000 vestibular schwannomas (acoustic neuromas): surgical management and results with an emphasis on complications and how to avoid them. Neurosurgery. 1997;40(1):11-23.

15. Briggs RJS, Luxford WM, Atkins JS, Hitselberger WE. Translabyrinthine removal of large acoustic neuromas. Neurosurgery. 1994;34(5):785-91.

16. Koos WT, Day JD, Matula C, Levy DI. Neurotopographic considerations in the microsurgical treatment of small acoustic neurinomas. J Neurosurg. 1998;88(3):506-12.

17. Tonn JC, Schlake HP, Goldbrunner R, Milewski C, Helms J, Roosen K. Acoustic neuroma surgery as an interdisciplinary approach: A neurosurgical series of 508 patients. J Neurol Neurosurg Psychiatry. 2000;69(2):161-6.

18. Zentner J, Albrecht $\mathrm{T}$, Hassler $\mathrm{W}$. Prevention of an air embolism by moderate hypoventilation during surgery in the sitting position. Neurosurgery. 1991;28(5):705.

19. Duke DA, Lynch JJ, Harner SG, Faust RJ, Ebersold MJ. Venous air embolism in sitting and supine patients undergoing vestibular schwannoma resection. Neurosurgery. 1998;42(6):1282-7.

20. Mavarez-Martinez A, Israelyan LA, Soghomonyan S, Fiorda-Diaz J, Sandhu G, Shimansky VN, et al. The Effects of Patient Positioning on the Outcome During Posterior Cranial Fossa and Pineal Region Surgery. Front Surg. 2020;7.

21. Fukuda M, Saito A, Takao T, Hiraishi T, Yajima N, Fujii Y. Drainage patterns of the superficial middle cerebral vein: Effects on perioperative managements of petroclival meningioma. Surg Neurol Int. 2015;6(1).

22. Yamakami I, Uchino Y, Kobayashi E, Yamaura A, Oka N. Removal of large acoustic neurinomas (vestibular schwannomas) by the retrosigmoid approach with no mortality and minimal morbidity. 2004;75(3):453-8.

23. Grant GA, Rostomily RR, Kim DK, Mayberg MR, Farrell D, Avellino A, et al. Delayed facial palsy after resection of vestibular schwannoma. J Neurosurg. 2002;97(1):93-6.

24. Lanman TH, Brackmann DE, Hitselberger WE, Subin B. Report of 190 consecutive cases of large acoustic tumors (vestibular schwannoma) removed via the translabyrinthine approach. J Neurosurg. 1999;90(4):617-23.

25. Sluyter S, Graamans K, Tulleken CAF, Van Veelen CWM. Analysis of the results obtained in 120 patients with large acoustic neuromas surgically treated via the translabyrinthinetranstentorial approach. J Neurosurg. 2001;94(1):61-6.

26. Mamikoglu B, Wiet RJ, Esquivel CR. Translabyrinthine Approach for the 
Management of Large and Giant Vestibular Schwannomas. Otol Neurotol. 2002;23(2):224-7.

27. Ebersold MJ, Harner SG, Beatty CW, Harper CM, Quast LM. Current results of the retrosigmoid approach to acoustic neurinoma. J Neurosurg. 1992;76(6):901-9.

28. Sterkers JM, Morrison GAJ, Sterkers O, el-Dine MMKB. Preservation of Facial, Cochlear, and other Nerve Functions in Acoustic Neuroma Treatment. Otolaryngol Neck Surg. 1994;110(2):146-55.
29. Gidley PW, Maw J, Gantz B, Kaylie D, Lambert $\mathrm{P}$, Malekzadeh $\mathrm{S}$, et al. Contemporary Opinions on Intraoperative Facial Nerve Monitoring. OTO open. 2018;2(3).

30. Starnoni D, Giammattei L, Cossu G, Link MJ, Roche PH, Chacko AG, et al. Surgical management for large vestibular schwannomas: a systematic review, meta-analysis, and consensus statement on behalf of the EANS skull base section. Vol. 162, Acta Neurochirurgica. Springer; 2020. 2595 617. 